\title{
Atomic Nitrogen Density Measurements in a Nanosecond Capillary Discharge
}

\author{
Tat Loon Chng, ${ }^{1}$ Nikita Lepikhin ${ }^{2}$ and Inna Orel ${ }^{3}$ \\ CNRS, Ecole Polytechnique, Sorbonne University, University Paris-Sud, Observatoire de Paris, University \\ Paris-Saclay, Palaiseau, 91128, France \\ Nikolay A Popov ${ }^{4}$ \\ Skobeltsyn Institute of Nuclear Physics, Moscow State University, Moscow, 119991, Leninsky gory, Russia \\ Svetlana Starikovskaia ${ }^{5}$ \\ CNRS, Ecole Polytechnique, Sorbonne University, University Paris-Sud, Observatoire de Paris, University \\ Paris-Saclay, Palaiseau, 91128, France
}

\begin{abstract}
This paper forms part of a larger study geared towards examining the atomic nitrogen production as a function of reduced electric fields, particularly in nanosecond type discharges. In this study, the atomic nitrogen density in a pure molecular nitrogen capillary discharge at 27 mbar is measured using two-photon absorption laser induced fluorescence (TALIF). Due to its small volume, the capillary provides an ideal geometry for achieving high levels of specific deposited energy into the plasma ( $>1 \mathrm{eV}$ per molecule) as well as high peak reduced electric fields ( $>500 \mathrm{Td}$ ). Under these conditions, our results show that unusually high densities of atomic nitrogen are recorded, with the time evolution measurements indicating a peak dissociation fraction of slightly more than $10 \%$. Further validation of these results with numerical simulations are ongoing, but on the current basis, support the notion that the capillary discharge is an extremely efficient configuration for the generation of nitrogen atoms.
\end{abstract}

\section{Introduction}

$\mathrm{O}$ ver the past two decades, the use of high voltage pulses occurring on the nanosecond timescale for plasma initiation has found broad applicability in diverse fields such as medicine, aerodynamics, combustion and even agriculture. Also commonly known as nanosecond discharges, the attractiveness of this enabling technology lies in its ability to generate higher than usual electric fields (or reduced electric fields) which can in turn alter the chemical reactivity of a particular system. This change in reactivity is expedited by the opportunity to couple energy into internal degrees of freedom of atoms or molecules, thereby leading to an extraneous production of reactive species such as electrons, ions, radicals and excited state particles, through processes such as electronic excitation, dissociation and ionization. As a consequence, much of recent research has provided insight into the transient chemical pathways made available by this infusion of reactive species. However, even under these conditions of high electric fields, an important distinction which should be made is the specific delivered energy to the discharge, i.e. energy normalized by the number of particles. When this number is low (nominally defined in terms of eV per molecule and abbreviated as $\mathrm{eV} / \mathrm{mol}$ ), the plasma kinetics involve mainly chemical reactions between the reactive species and the background/bath gas. At increased specific delivered energies (on the order of $1 \mathrm{eV} / \mathrm{mol}$ ), which is the focus of this study, the probability of interaction between reactive species becomes much likelier.

The primary objective of this work is to experimentally measure the atomic nitrogen $(\mathrm{N})$ production in a low pressure, molecular nitrogen $\left(\mathrm{N}_{2}\right)$ capillary discharge using two-photon laser induced fluorescence (TALIF). Due to its unique geometry (small diameter and volume), a cylindrical capillary tube provides an ideal configuration for producing nanosecond discharges with high values of specific delivered energy. The natural idea is to make use of these measurements to aid in the modeling efforts of such discharges. The current study complements

\footnotetext{
${ }^{1}$ Postdoctoral researcher, Laboratory of Plasma Physics.

${ }^{2}$ Postdoctoral researcher (former), Laboratory of Plasma Physics.

${ }^{3}$ Graduate student, Laboratory of Plasma Physics.

${ }^{4}$ Leading scientific researcher, Skobeltsyn Institute of Nuclear Physics.

${ }^{5}$ Senior researcher, Laboratory of Plasma Physics.
} 
previous work, in which important aspects of this discharge such as the delivered energy, electric field and temperature have been quantified [1]. In addition, similar measurements of atomic oxygen (O) have also been performed using an identical geometry [2].

\section{Experimental Setup}

A schematic of the discharge cell geometry used in this work is presented in Fig. 1. This configuration is very similar to that used in Ref. [1]. A cylindrical quartz capillary with a nominal length of $67 \mathrm{~mm}$ and an internal and external diameter of $1.7 \mathrm{~mm}$ and $3.0 \mathrm{~mm}$ respectively is placed between two pin-shaped electrodes and encapsulated by a rectangular $48 \mathrm{~mm}$ by $67 \mathrm{~mm}$ metallic screen. Pure $\mathrm{N}_{2}$ gas enters and exits the capillary via two glass side arms at a flow rate of $5 \mathrm{sccm}$ by means of a Brooks Instruments 5850TR mass flow controller. This flow rate was chosen with a view to ensuring gas renewal before each successive high voltage pulse. The static pressure in the capillary was kept constant at 27 mbar based on previous work which indicated this as a reasonable pressure for initiating a reproducible and homogeneous plasma. Pressure measurements are made using a pair of CMR 362 capacitive pressure sensors (from Pfeiffer Vacuum SAS), each positioned at an approximately equidistant location upstream and downstream of the capillary. The operating pressure was determined by taking the average of both these sensor readings. This approach for pressure quantification was chosen due to difficulties associated with mounting a pressure transducer directly onto the capillary and was validated numerically using a Navier-Stokes solver [3].

High voltage nanosecond pulses are delivered by means of a FPG 12-1NM high-voltage generator (HVG) manufactured by FID GmbH. Positive polarity, $9.3 \mathrm{kV}$ amplitude voltage waveforms with a rise time of $4 \mathrm{~ns}$ and a full width at half maximum (FWHM) of $30 \mathrm{~ns}$ are produced by this high voltage source at a repetition rate of 10 $\mathrm{Hz}$. The output of this high-voltage generator is connected to one of the electrodes (henceforth termed highvoltage electrode) via a 50 ohm impedance RG213 coaxial cable. A second such coaxial cable of identical length is connected to the remaining electrode (low-voltage electrode) but is kept ungrounded at a floating potential. The shielding of both these coaxial cables is connected to the rectangular metallic screen. Due to an impedance mismatch between the high voltage pulses and the discharge (viz. electrical load), each pulse delivered by the HVG is reflected twice, once from the high-voltage electrode and a second time from the HVG itself. This leads to a sequence of 3 incident pulses with gradually diminishing voltage seen by the discharge, each separated by about 250 ns. Custom-made, calibrated back current shunts are installed at mid-length of both coaxial cables for the purpose of measuring the specific energy coupled to the plasma.

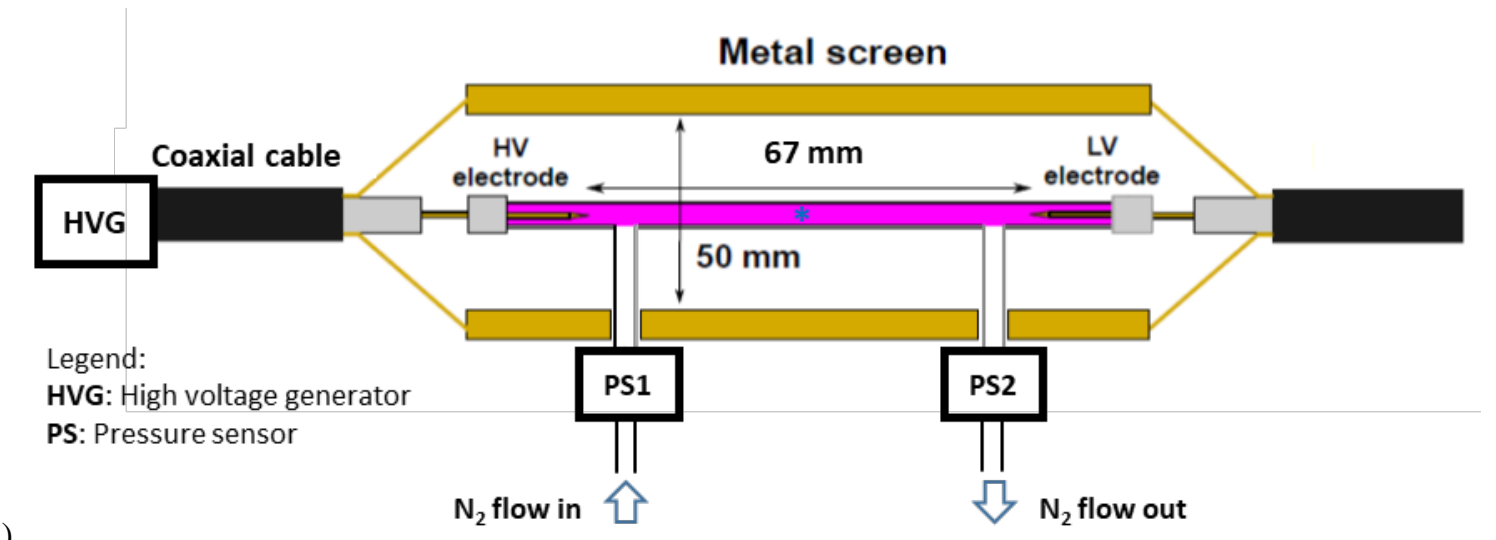

a)

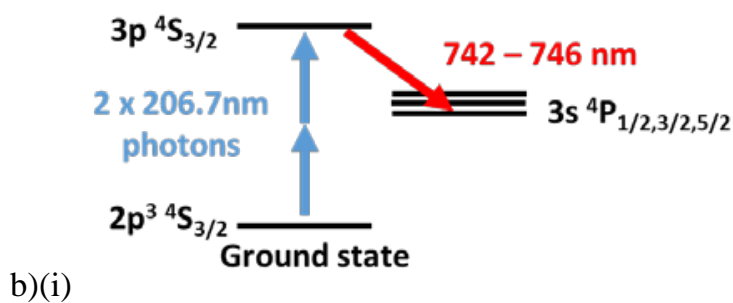

(ii)

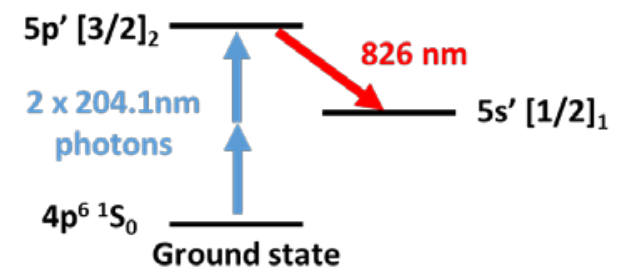

Fig. 1 a) Schematic of the discharge cell setup used in this study. (The region probed by the TALIF measurements is marked with a blue asterisk.) b) (i) $\mathrm{N}$ and (ii) Kr TALIF scheme.

The laser system used for the TALIF experiments consists of a Sirah Lasertechnik Cobra Stretch dual-grating dye laser driven by a frequency-doubled Quanta Ray Nd:YAG laser. A dye-mix of Rhodamine B and Rhodamine 101 (supplied by Sirah Lasertechnik) is employed as the lasing medium, and when pumped with the required 532 $\mathrm{nm}$ light, produces output within the desired visible range of $621 \mathrm{~nm}$ at a repetition rate of $10 \mathrm{~Hz}$. This light is 
then frequency-tripled via two successive alpha-BBO non-linear crystals so as to yield $3 \mathrm{~mJ}, 207 \mathrm{~nm}$ pulses (at the same repetition rate) with a pulse duration of about $6.5 \mathrm{~ns}$. This UV light is focused with a 500 mm planoconvex lens, propagating along an axis orthogonal to the longitudinal axis of the capillary. As illustrated in Fig. 1a), care was taken to ensure that the laser beam probed a region corresponding to the center of the capillary. To avoid damaging the capillary, the laser intensity was attenuated by locating the beam focus slightly downstream of the capillary tube.

The N TALIF scheme used for these experiments is shown in Fig. 1b)(i). The two-photon $3 \mathrm{p}^{4} \mathrm{~S}_{3 / 2} \mathrm{~N}$ resonance is accessed via a pair of $206.7 \mathrm{~nm}$ photons and subsequently fluoresces to three different fine structure levels, emitting light respectively at $742.6 \mathrm{~nm}, 744.4 \mathrm{~nm}$ and $747.0 \mathrm{~nm}$ [4]. For the majority of the experiments, this resulting fluorescence is captured by a Hamamatsu photomultiplier (Photo Sensor Module H7422P) with an attached Thorlabs FB750-10 bandpass filter (center wavelength 750 nm, 10 nm FWHM). Signals are obtained by averaging over 400 laser shots within a laser intensity fluctuation of $\pm 3 \%$. An IR-sensitive Andor Technology SR-500i-B1 spectrometer with an attached ICCD camera was used to resolve and observe the fluorescence spectra.

In order to obtain quantitative $\mathrm{N}$ densities, signal calibration against an inert, reference gas - in this case krypton - was performed. This procedure - first described in seminal work by [5] - has since been widely adopted in the literature and essentially makes use of the fluorescence from a gas of known density (in this case $\mathrm{Kr}$ ) to calibrate the signal obtained from a gas of unknown density $(\mathrm{N})$. This approach circumvents the need for producing a known amount of $\mathrm{N}$ which can often be a less than straightforward task. The corresponding $\mathrm{Kr}$ TALIF scheme involves pumping the 5p' $[3 / 2]_{2}$ resonance with two photons at $204.1 \mathrm{~nm}$, followed by fluorescence to the $5 s^{\prime}[1 / 2]_{1}$ state at $826 \mathrm{~nm}$ (see Fig. 1b)(ii)).

One advantage of the above approach which is relevant to this study is that the non-uniform laser intensity distribution produced by locating the beam focus away from the measurement volume (i.e. capillary) is identically replicated, and thus accounted for, in the Kr calibration experiment.

Based on [5], the number density of atomic nitrogen may be written as:

$$
n_{N}=\left(\frac{\sigma_{K r}}{\sigma_{N}}\right)\left(\frac{v_{N}}{v_{K r}}\right)^{2}\left(\frac{P M T_{K r}}{P M T_{N}}\right)\left(\frac{A_{K r}}{A_{N}}\right)\left(\frac{F_{K r}}{F_{N}}\right)\left(\frac{A_{N}+Q_{N}}{A_{K r}+Q_{K r}}\right)\left(\frac{I_{K r}}{I_{N}}\right)^{2}\left(\frac{S_{N}}{S_{K r}}\right) n_{K r}
$$

where $n$ is the number density, $\sigma$ is the two-photon absorption cross-section, $v$ is the resonance frequency, $P M T$ is the photomultiplier sensitivity at the resonant wavelength(s), $A$ is the natural radiative rate, $F$ is the filter transmission, $Q$ is the quenching rate of the two-photon excited state, $I$ is the laser intensity and $S$ is the spectrally and temporally integrated fluorescence measured by the photomultiplier. Subscripts $\mathrm{N}$ and Kr refer to atomic nitrogen and krypton respectively. The ratio of two-photon cross-sections (value of 0.67) is obtained from [5]; PMT sensitivities and filter transmission curves are obtained or extrapolated from their respective manuals. The natural radiative rate of $\mathrm{N}$ (value of $29.6 \mathrm{~ns}$ ) is obtained from [5], while the Kr number density of $6.7 \times 10^{16} \mathrm{~cm}^{-3}$ is obtained by flowing $\mathrm{Kr}$ gas at room temperature through the capillary at a pressure of 2.7 mbar.

In order to ensure operation within the 'linear' regime, defined as when the fluorescence signal $S$ observes a quadratic dependence on the laser intensity (see eqn. (1)), the laser energy was varied over a range of 10-300 $\mu \mathrm{J}$ in order to establish the optimum laser intensity. The energy used for the $\mathrm{N}$ and Kr experiments were $85 \mu \mathrm{J}$ and $30 \mu \mathrm{J}$ respectively.

\section{Results and Discussion}

The evolution of the $\mathrm{N}$ atom density at various time instants with respect to the discharge initiation is shown in Fig. 2. These measurements are obtained by integrating the resonant fluorescence signals captured by the photomultiplier (PMT) for both Kr and N, followed by application into equation (1). The laser was tuned off resonance in order to subtract the undesired fluorescence from the quartz capillary as well as overlapping fluorescence from the background plasma emission. This background emission, which was observed to last for about $1 \mu \mathrm{s}$, meant that measurements made during or before this time were plagued by a much poorer signal to noise ratio. Where possible, the data shown in Fig. 2 are corrected for excited state quenching; quenching rates are determined by fitting an exponential function to the decaying section of the PMT signal. Two distinctive features of this temporal behavior are worthy of mention. Firstly, the $\mathrm{N}$ atom density is observed to be relatively long-lived (especially in comparison with the timescale of the nanosecond excitation) - measurable N TALIF signals are evident even up to $10 \mathrm{~ms}$ after the discharge is initiated. At these longer timescales, it is believed that the main loss mechanisms for atomic nitrogen are wall recombination and atom-atom recombination. For an $\mathrm{N}$ density of about $10^{17} \mathrm{~cm}^{-3}$, based on rate coefficients obtained in [6] and [7], the atom-atom recombination time is estimated to be about $10 \mathrm{~ms}$ (for a relevant rate coefficient of $k \approx 10^{-33} \mathrm{~cm}^{6} \mathrm{~s}^{-1}$ ) while the wall recombination time is estimated to be between $1-10 \mathrm{~ms}$ for a temperature range of 300 to $2000 \mathrm{~K}$. The characteristic wall recombination time is estimated based on $\tau_{w r}=D /\left(v_{a v} \gamma\right)$ where $D$ is the pressure-dependent diffusion coefficient, $v_{a v}$ is the mean kinetic velocity of particles and $\gamma$ is the recombination probability for atomic nitrogen with quartz. Previous measurements have found that the gas temperature rises from $300 \mathrm{~K}$ to about $2100 \mathrm{~K}$ from the point of 
initiation till around $1.5 \mu \mathrm{s}$ in the near afterglow. Both these estimates of the limiting recombination times seem reasonable when compared with the data. A direct consequence of this finding is that attempts to model nanosecond discharges with repetition rates faster than $10 \mathrm{~ms}$ (e.g. $\mathrm{kHz}$ or $1 \mathrm{~ms}$ ) should take into account the residual atomic species production from previous pulses.

At earlier times, the other timescales of interest are the acoustic timescale and the heat diffusion time to the wall. The acoustic timescale may be simplistically defined as half the length of the capillary divided by the temperature-dependent speed of sound. Based on an earlier study using a similar discharge geometry, this figure was estimated to be about $40 \mu \mathrm{s}$. The acoustic time essentially dictates when the pressure within the capillary equilibrates and returns to its initial value of 27 mbar. Prior to this time, an isochoric or constant density approximation may be assumed. In addition, it is found that the heat conduction time to the wall is estimated to be on the order of about $5 \mu \mathrm{s}$. The heat diffusion timescale is defined as $\tau_{\text {diff }}=(r / 2.4)^{2} / D$ where $r$ is the radius of the capillary and the value of 2.4 may be treated as a shape factor for a cylindrical geometry. Since the heat diffusion timescale is faster than the acoustic timescale, this provides one of the possible reasons why there is no significant change in the $\mathrm{N}$ density (due to the gas-dynamic rarefaction of the hot channel) after the discharge.

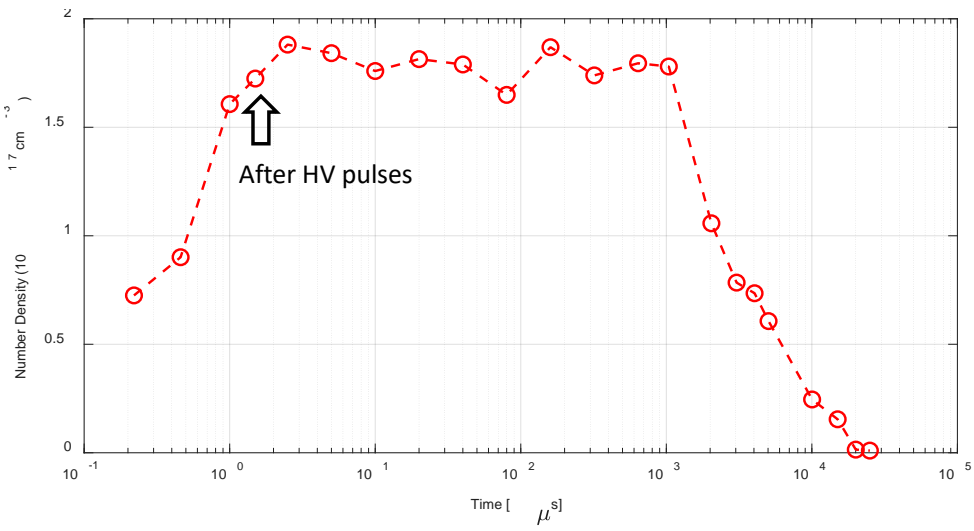

Fig. 2 Temporal evolution of $\mathbf{N}$ atom density.

The second feature of note is the fact that the TALIF signals recorded within the first few microseconds after the discharge exhibit symptoms of strong quenching, as evidenced by the fast decay rates of the attendant PMT signals similar to an example displayed in Fig. 3.

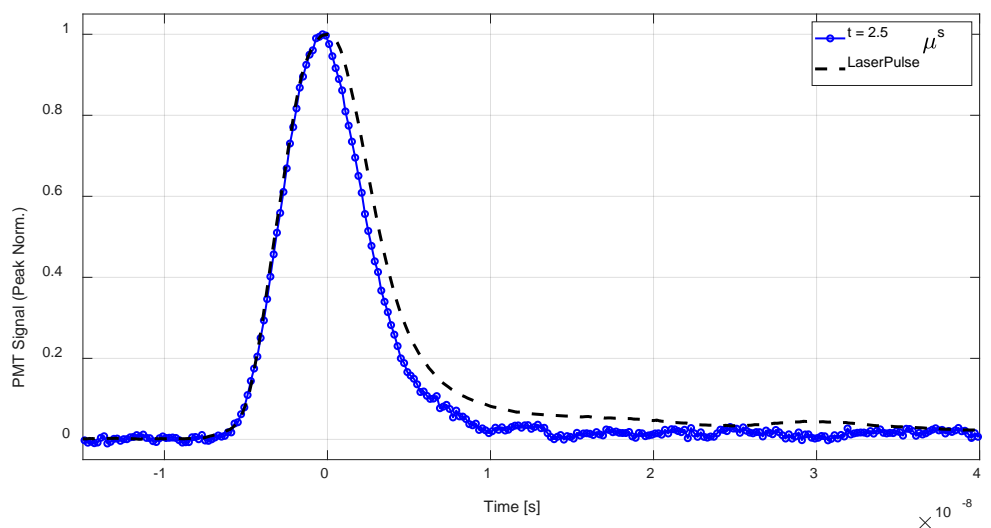

Fig. 3 Raw PMT resonant time trace (400 shot averaged) versus temporal profile of laser intensity acquired from a 1 ns response Thorlabs DET10A photodiode.

Quenching corrections cannot be applied in this regime since the decay rates are limited by the duration of the laser pulse and therefore renders the actual quenching rate unknown. This point becomes clearer in the context of Fig. 4, which plots the measured TALIF signal decay rates (in inverse ns) as the same function of time shown in Fig. 2. The exact origin of this quenching remains unclear, but could be due to either electrons, self-quenching from $\mathrm{N}$, or hot $\mathrm{N}_{2}$ molecules produced by fast gas heating [1]. Evidence of self-quenching - due to the large density of atomic nitrogen produced - may be seen from the fact that even at the millisecond timescale (when the temperature may be assumed to be close to that of the ambient and $\mathrm{N}_{2}$ is expected to be the main quenching partner), the decay rates still slightly exceed that predicted by considering room temperature $\mathrm{N}_{2}$ quenching rates [5]. 


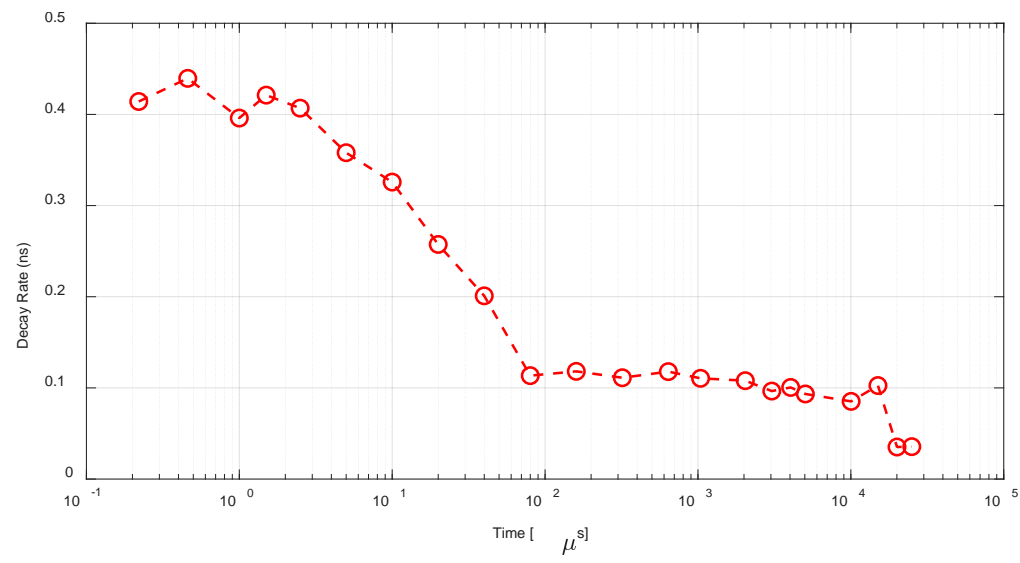

Fig. 4 Temporal evolution of the TALIF signal decay rates. Signal lifetime is laser-pulse limited up to about $3 \mu$ s and therefore quenching corrections are only applicable beyond that time.

Due to non-uniform transmission of the bandpass filter within the wavelength vicinity of the $\mathrm{N}$ fluorescence (742 nm to $747 \mathrm{~nm}$ ), there was a need to verify the linestrengths of the three respective transitions (see Fig. 1b)). Spectrally resolved emission spectra displayed in Fig. 5 shows excellent agreement with the linestrengths provided in [4].

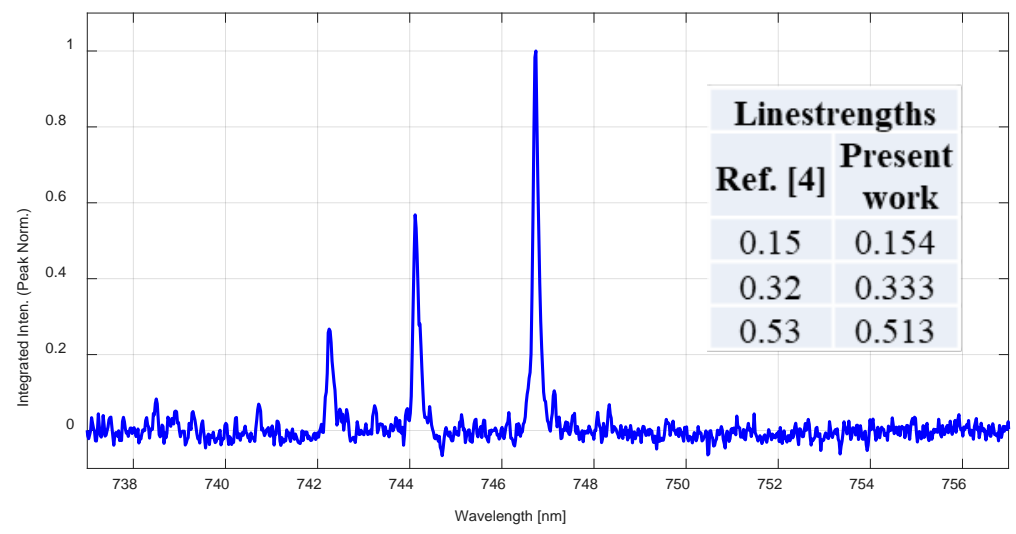

Fig. $5 \mathrm{~N}$ atom fluorescence spectra recorded by averaging over 1000 laser shots. Experimental linestrengths are computed by taking the spectral integral of each line normalized by the total spectral integral.

The peak value of the measured $\mathrm{N}$ density in Fig. 2 of about $1.5 \times 10^{17} \mathrm{~cm}^{-3}$ represents a dissociation degree of about $11 \%$. Here the dissociation degree is defined as $n_{N} / 2 n_{N 2}$ and provides an estimate of the fraction of the total number of $\mathrm{N}_{2}$ molecules which have been converted into their atomic constituents. As a sanity check of this unusually large number, a simple specific energy calculation based on various dominant processes is carried out using known conditions of the discharge at $1.5 \mu$ s. These dominant processes include $\mathrm{N}$ atom dissociation, $\mathrm{N}$ and $\mathrm{N}_{2}$ gas heating, as well as $\mathrm{N}_{2}$ vibrational mode heating. The idea is to compare the energy required for these processes against the measured specific energy deposited into the plasma. The latter was computed based on the back current shunt (BCS) data for the $3 \mathrm{HV}$ pulses following a well-established procedure [1]. This value for the total specific energy is about $1.8 \mathrm{eV}$ per molecule. As one might expect, most of the energy is deposited in the first pulse when the plasma is formed together with the passage of the fast ionization wave and the discharge gap is 'closed'. As an example, the specific deposited energy in the first pulse is shown in Fig. 6.

For a dissociation fraction of $11 \%$, Fig. 7 indicates that the total energy required for the processes of dissociation and heating described above is about $1.7 \mathrm{eV}$. This value is relatively close but still less than the measured value of $1.8 \mathrm{eV}$ which suggests that the data from the $\mathrm{N}$ density experiments lies within physical limits. Nonetheless, it is acknowledged that these measurements do appear unusually large and warrant further examination. If indeed valid, this could signify the existence of an extremely efficient $\mathrm{N}$ atom dissociation mechanism related to electron impact dissociation from the A, B and C electronically excited states of $\mathrm{N}_{2}$. Estimates of these cross-sections have been provided in [8]. 


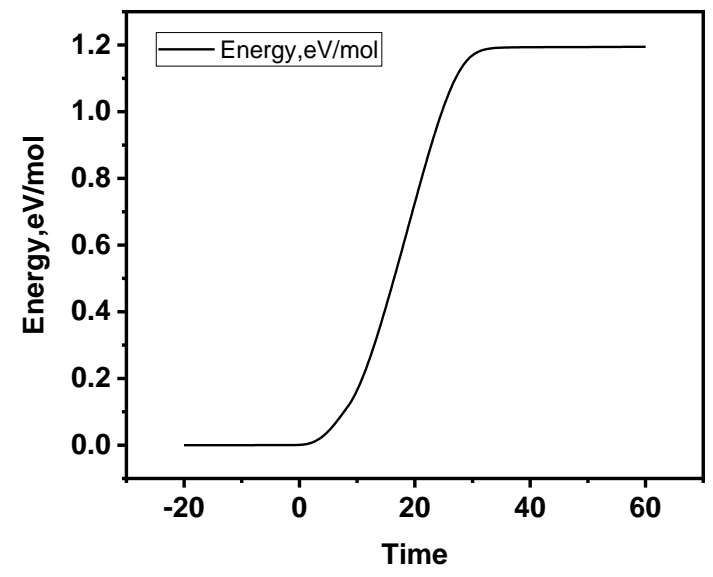

Fig. 6 Specific deposited energy during the first high voltage pulse $(\approx 1.2 \mathrm{eV} / \mathrm{molecule})$ computed from the BCS data following a similar procedure as in [1].

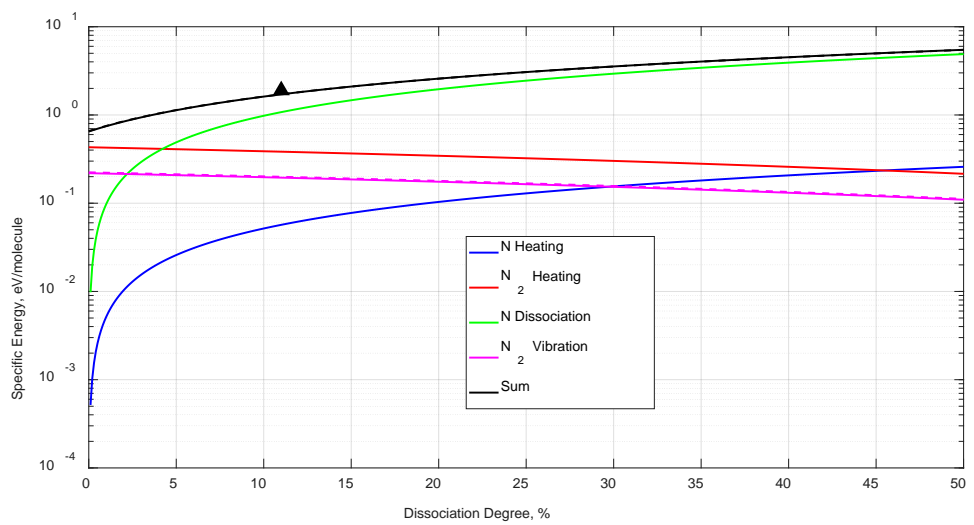

Fig. 7 Specific energy required for various different processes as a function of dissociation degree. The measured specific energy delivered to the discharge is indicated with a filled black triangle.

\section{Conclusions and Future Work}

The $\mathrm{N}$ atom production in a pure $\mathrm{N}_{2}$ nanosecond capillary discharge at 27 mbar has been successfully measured using TALIF. The results reveal $\mathrm{N}$ atom densities on the order of about $10^{17} \mathrm{~cm}-3$, representative of a peak dissociation fraction of about $11 \%$. While these values may seem unusually high, estimates of the characteristic timescales of the problem as well as the specific deposited energy appear to support these large $\mathrm{N}$ atom densities as well as its consequent temporal evolution. Further work is ongoing, particular with regards to marrying the experimental data with a well-established plasma kinetic model. The axial distribution of $\mathrm{N}$ atom density as well as different gas compositions is also a subject of future research.

\section{Acknowledgments}

This work is partially supported by the French National Research Agency, ANR (ASPEN Project), LabEx Plas@Par and the French-Russian international laboratory LIA KaPPA “Kinetics and physics of pulsed plasmas and their afterglow (including RFBR project 17-52-16001 and CNRS financial and organization support)”.

\section{References}

[1] Lepikhin, N. D., Popov, N. A., \& Starikovskaia, S. M. (2018). Fast gas heating and radial distribution of active species in nanosecond capillary discharge in pure nitrogen and N2: O2 mixtures. Plasma Sources Science and Technology, 27(5), 055005.

[2] Klochko, A. V., Lemainque, J., Booth, J. P., \& Starikovskaia, S. M. (2015). TALIF measurements of oxygen atom density in the afterglow of a capillary nanosecond discharge. Plasma Sources Science and Technology, 24(2), 025010. 
[3] Klochko A 2014 Excited species chemistry in homogeneous nanosecond discharges with high specific energy deposition. Ph.D. thesis Ecole Polytechnique.

[4] Kramida, A., Ralchenko, Yu., Reader, J. and NIST ASD Team (2018). NIST Atomic Spectra Database (version 5.5.6).

[5] Niemi, K., Schulz-Von Der Gathen, V., \& Döbele, H. F. (2001). Absolute calibration of atomic density measurements by laser-induced fluorescence spectroscopy with two-photon excitation. Journal of Physics D: Applied Physics, 34(15), 2330.

[6] Evenson, K. M., \& Burch, D. S. (1966). Atomic-nitrogen recombination. The Journal of Chemical Physics, 45(7), 24502460

[7] Kim, Y. C., \& Boudant, M. (1991). recombination of O, N, and H atoms as Silica: Kinetics and mechanism, hagemuir, 7, 2999-3005 (1991)

[8] Bacri, J., \& Medani, A. (1980). Electron diatomic molecule weighted total cross section calculation: II. Application to the nitrogen molecule. Physica B+ C, 101(3), 410-419. 